\title{
Early Machine-Human Interface around Sepsis Severity Identification: From Diagnosis to Improved Management?
}

\author{
Vikas Bansal ${ }^{1}$, Emir Festić ${ }^{1}$, Muhammad A. Mangi ${ }^{1}$, Nicholl A. Decicco ${ }^{2}$, Ashley N. \\ Reid $^{2}$, Elizabeth L. Gatch ${ }^{2}$, James M. Naessens ${ }^{3,4}$ Pablo Moreno-Franco ${ }^{5}$
}

${ }^{1}$ Department Critical Care Medicine, Mayo Clinic, Jacksonville, FL, USA ${ }^{2}$ Department of Emergency Medicine, Mayo Clinic, Jacksonville, FL, USA ${ }^{3}$ Center for the Science of Healthcare Delivery, Mayo Clinic, Jacksonville, FL, USA, ${ }^{4}$ Health Care Policy and Research, Mayo Clinic, Rochester, MN, USA ${ }^{5}$ Department of Transplant Critical Care Medicine, Mayo Clinic, Jacksonville, FL, USA

\author{
Correspondence: \\ MorenoFranco.Pablo@mayo.edu \\ Tel.: + 19049563298 \\ Fax: + 19049563262
}

Received: 5 October 2017

Accepted: 31 May 2018

Key words: Computerized decision support - Sepsis - Algorithm.

\begin{abstract}
Objective. To investigate the statistical measures of the performance of 2 interventions: a) early sepsis identification by a computerized sepsis "sniffer" algorithm (CSSA) in the emergency department (ED) and b) human decision to activate a multidisciplinary early resuscitation sepsis and shock response team (SSRT). Methods. This study used a prospective and historical cohort study design to evaluate the performance of two interventions. Intervention. A computerized sepsis sniffer algorithm (CSSA) to aid in early diagnosis and a multidisciplinary sepsis and shock response team (SSRT) to improve patient care by increasing compliance with Surviving Sepsis Campaign (SSC) bundles. Results. The CSSA yielded a sensitivity of 100\% (95\% CI, $99.13-100 \%)$ and a specificity of $96.2 \%$ (95\% CI, $95.55-96.45 \%)$ to identifying sepsis in the ED (Table 1). The SSRT resource was activated appropriately in $34.1 \%(86 / 252)$ of patients meeting severe sepsis or septic shock criteria; the SSRT was inappropriately activated only three times in sepsis-only patients. In 53\% (134/252) of cases meeting criteria for SSRT activation, the critical care team was consulted as opposed to activating the SSRT resource. Conclusion: Our two-step machine-human interface approach to patients with sepsis utilized an outstandingly sensitive and specific electronic tool followed by more specific human decision-making.
\end{abstract}

\section{Introduction}

Approximately 750,000 patients develop severe sepsis and septic shock in the United States each year (1). More than half of them are admitted to an intensive care unit (ICU) accounting for $51 \%$ of all ICU admissions, 20-30\% of hospital deaths, and $\$ 15.4$ billion in annual health care costs (1-3) Early diagnosis and treatment of these patients is critical and is associated not only with improved morbidity and mortality, but also reduced length of stay (LOS) (4-7). After the landmark randomized control trial by Rivers et al
(2001) endorsing early goal directed therapy (EGDT), institutions around the world have developed, implemented and revised quality improvement (QI) initiatives to incorporate EGDT into clinical practice (4).

The delayed diagnosis of sepsis coupled with the lack of reliable rule-out criteria in the emergency department (ED) has been associated with high mortality risk, which dictates improvements in the timeliness of sepsis detection by developing an automated system (8-11). The available published literature regarding systems within Electronic 
health records (EHR) to improve early identification of septic patients substantially differed in triggering criteria, effector responses, and study settings. Results of the studies investigating the effect of computerized monitoring in addition to response in the non-ICU acute inpatient setting endorsed an improvement in the timeliness of diagnostic and therapeutic interventions (11), but have not significantly affected patient outcomes (8). This may be due to inadequacies in triggering criteria or inadequacies in response to the alerts. Overall, the reported sensitivity and specificity in previous studies on computerized algorithms for sepsis identification remained unsatisfactory $(12,13)$. The team approach to sepsis care has proven effective as evidenced by improved bundle compliance and patient outcomes. A prospective study analyzed outcomes following implementation of standardized interdisciplinary order sets for patients with severe sepsis/septic shock. Sepsis order sets implemented using the Six-Source Influencer Model to create an atmosphere of teamwork to help counteract resistance to change, improved the process of care (14). A more recent study published in 2016 evaluated the effect of a QI sepsis management bundle on mortality and sepsis protocol compliance, determining that interdisciplinary ED sepsis bundles composed of triage flagging, registered nurse $(\mathrm{RN})$ medical directives, education campaigns, and modified sepsis protocols improved early diagnosis and protocolized medical care resulting in improve care (15).

In line with the literature, Mayo Clinic Florida (MCF) employed multiple measures to improve the care of the septic patient, specifically in the emergency department (ED). A computerized sepsis sniffer algorithm (CSSA) to aid in early diagnosis and a multidisciplinary sepsis and shock response team (SSRT) to improve patient care by increasing compliance with the Society of Critical Care Medicine (SCCM) bundles was deployed in September 2013 as part of a QI project already in place (16). Examination of these QI efforts over a period of three months revealed a low observed-to-expected incidence of sepsis, prompting this QI effort. MCF's CSSA was revamped multiple times, aiming to find the appropriate degrees of sensitivity and specificity. While there is research on computerized algorithms to diagnose sepsis and the team approach to the septic patient, there is a dearth of quantitative research related to the human response to these methods.

Therefore, the purpose of this study was to: (1) evaluate the effectiveness of the CSSA, and (2) evaluate the human decision to activate the SSRT once computerized algorithms recognize a patient meeting sepsis criteria.

\section{Methods}

The cyber-realm of caring for the septic patient at MCF starts when a computer algorithm recognizes a patient with systemic inflammatory response syndrome (SIRS) or sepsis based upon qualifying criteria. Once triggered, the computer algorithm alerts the ED team lead nurse who notifies the ED provider that the patient met criteria for either SIRS or sepsis. The ED physician then evaluates the patient and confirms whether the patient is actually septic. The ED nursing staff utilizes a checklist to promote completion of all bundle requirements.

In addition to the computer algorithm, MCF also developed a multidisciplinary Sepsis and Shock Response Team (SSRT) composed of an ICU physician, an ICU fellow or resident, an advanced practice provider (APP), a nursing supervisor, and a pharmacist. Once the ED provider confirms the patient has either severe sepsis or septic shock, the SSRT is activated and the team is expected to report to the ED within 
15 minutes to evaluate the patient, encourage completion of bundle requirements, and collaborate with ED providers to triage the patient to the appropriate level of inpatient care. The SSRT is not to be activated for sepsis only patients. Using multiple PlanDo-Study-Act (PDSA) cycles, the QI team at MCF tracks bundle compliance, provides feedback to providers at monthly staff meetings, and communicates feedback via biweekly e-mails.

\section{Study design}

This study used a prospective and historical cohort study design to evaluate the performance of two interventions. The study proposal was approved by the Mayo Clinic Institutional Review Board (IRB) as a QI project. Review of the electronic medical record (EMR) was done with pre-obtained patient consent.

\section{Study population}

To have an accurate assessment of the CSSA, our study included all ED patients who had sepsis sniffer alerts and SSRT activations with a final principal diagnosis of septicemia resulting in a diagnosis-related group (DRG) of septicemia or severe sepsis (MSDRGs 870-872) during the study period September $1^{\text {st }} 2013$ to August $31^{\text {st }} 2014$.

\section{Data collection}

Based on discharge DRGs of septicemia or severe sepsis, physician reviewers retrospectively abstracted patient demographic information, Acute Physiology and Chronic Health Evaluation (APACHE) IV scores, rates of ICU consultations, hospital length of stay (LOS), and mortality. Two physician reviewers (V. B. and M. M.) categorized patients based on standardized clinical definitions of sepsis, severe sepsis and septic shock, approved by Mayo Clinic enterprise subspecialty councils for the Departments of Emergency Medicine and Critical Care Medicine (Appendix 2). Reviewers were blinded to whether or not the CSSA was triggered or the SSRT was activated. Reviewers evaluated the cohort over two distinct time periods: the first four hours after ED presentation ( $0-4$ hours) and the subsequent 8 hours (4-12 hours) in order to assess if after initial management and triage, septic patients progressed to a more severe end of the sepsis spectrum and required escalation of care.

Three ED nurses (N.D.,A.R., and E.G.), part of a larger continuous QI initiative already in place, incorporated data on CSSA triggers and subsequent SSRT utilization in to the historic cohort based on DRGs. Additionally two ED nurses (N.D \& A.R.) retrospectively provided documented code status on admission.

\section{Statistical analysis}

Outcomes and variables were assessed using descriptive statistics. Continuous variables were reported using medians with the $1^{\text {st }}$ and $3^{\text {rd }}$ interquartile range (IQR). Sensitivity, specificity, false positive rate were calculated for the CSSA triggers. Sepsis and Shock Response Team utilization was reported as "appropriate" or "inappropriate" utilization. We first performed univariate analyses to determine the unadjusted associations of the SSRT activation and other predictor variables with the mortality due to sepsis on hospital discharge. We used nonparametric tests (Fisher's and Wilcoxon, as applicable) assuming the worst case scenario of nonnormalcy. A P value of less than 0.2 was considered statistically significant. Inclusion decision for P-value of 0.2 could be justified by the exploratory character of the multivariate analysis and by our intention to fare on the more conservative side. We excluded 
age from the multivariate logistic regression because of inclusion of APACHE-IV Score. The risk estimates were reported as odds ratios (OR) with 95\% confidence intervals (CI). The data was analyzed using the JMP ${ }^{\circledR}$ Pro 10.0.0 statistical software package (SAS Inc., Cary, NC, USA).

\section{Results}

A total of 27,106 patients presented to the ED between September 2013 and August 2014. A total of 1,431 patients triggered the CSSA within four hours of arrival to the ED. Of those CSSA triggers, 424 patients were deemed septic based on discharge DRGs. Upon manual review of the 424 patients, 419 were truly septic within 4 hours of presentation to the ED. Therefore, CSSA yielded a sensitivity of $100 \%$ (95\% CI, 99.12- 100.00\%) and a specificity of $96.21 \%$ (95\% CI, 95.97 $-96.43 \%)$ in identifying sepsis in the ED (Table 1). Thirty-four percent of the $252 \mathrm{pa}$ tients meeting criteria for severe sepsis or septic shock, as determined by retrospective review, had an appropriate SSRT activation. In 53\% (134/252) of cases meeting criteria for SSRT activation, the ICU was consulted directly as opposed to first activating the SSRT resource (Figure 1, Table 2 and Online supplemental material). The SSRT was inappropriately utilized only three times in sepsis-only patients.

In the initial phase (0-4 hours), 167 patients met criteria for sepsis-only, 176 met criteria for severe sepsis, and 76 met criteria for septic shock. Five patients were deemed not septic (Figure 1). In the second time period (hours 4-12), one patient progressed from sepsis-only to severe sepsis and two patients progressed from severe sepsis to septic shock. An ICU consult was obtained in one of two patients with septic shock. Of the five patients who did not meet sepsis criteria during the first four hours, over the following 8 hours (hours 5-12) one developed sepsis-only, two developed severe sepsis, one developed septic shock, and one remained sepsis-negative (Online supplemental material).

In univariate analysis, APACHE IV, full code status on admission, SSRT activation and ICU consultation were variables associated with in-hospital survival (Table 3a). In

Table 1. Screening performance characteristics for computerized sepsis sniffer algorithm in ED patients

\begin{tabular}{|c|c|c|c|c|c|c|}
\hline $\begin{array}{l}\text { Sepsis sniffer } \\
\text { performance }\end{array}$ & Sepsis & No sepsis & Total & Sensitivity & Specificity & FP rate \\
\hline Activation & 419 & 1012 & 1431 & \multirow{3}{*}{$\begin{array}{l}100.00 \% \\
(95 \% \text { Cl: } 99.12-100.00 \%)\end{array}$} & \multirow{3}{*}{$\begin{array}{l}96.21 \% \\
\text { (95\% Cl: } 95.97-96.43 \%)\end{array}$} & \multirow{3}{*}{$3.79 \%$} \\
\hline No activation & 0 & 25675 & 25675 & & & \\
\hline Totals & 419 & 26687 & 27106 & & & \\
\hline
\end{tabular}

$\mathrm{ED}=$ Emergency department; $\mathrm{Cl}=$ Confidence interval; $\mathrm{FP}=$ False positive rate

Table 2. Performance characteristics for human interface decision in severe sepsis or septic shock ED patients in the first 4 hours

\begin{tabular}{|c|c|c|c|c|c|}
\hline Intervention & $\begin{array}{l}\text { Severe sepsis or septic } \\
\text { shock }(n=252)\end{array}$ & $\begin{array}{l}\text { Sepsis only } \\
(n=167)\end{array}$ & $\begin{array}{l}\text { Total } \\
(n=419)\end{array}$ & $\begin{array}{l}\text { Appropriate } \\
\text { utilization }\end{array}$ & $\begin{array}{l}\text { Inappropriate } \\
\text { utilization }\end{array}$ \\
\hline SSRT activation & 86 & 3 & 89 & \multirow{2}{*}{$\begin{array}{l}34.1 \% \\
(86 / 252)\end{array}$} & \multirow{2}{*}{$\begin{array}{l}1.8 \% \\
(3 / 167)\end{array}$} \\
\hline No SSRT activation & 166 & 164 & 330 & & \\
\hline ICU consult & 134 & 19 & 153 & \multirow{2}{*}{$\begin{array}{l}53.2 \% \\
(134 / 252)\end{array}$} & \multirow{2}{*}{$\begin{array}{l}11.4 \% \\
(19 / 167)\end{array}$} \\
\hline No ICU Consult & 118 & 148 & 266 & & \\
\hline
\end{tabular}

$\mathrm{ED}=$ Emergency department; SSRT=Sepsis and shock response team; ICU=Intensive care unit. 


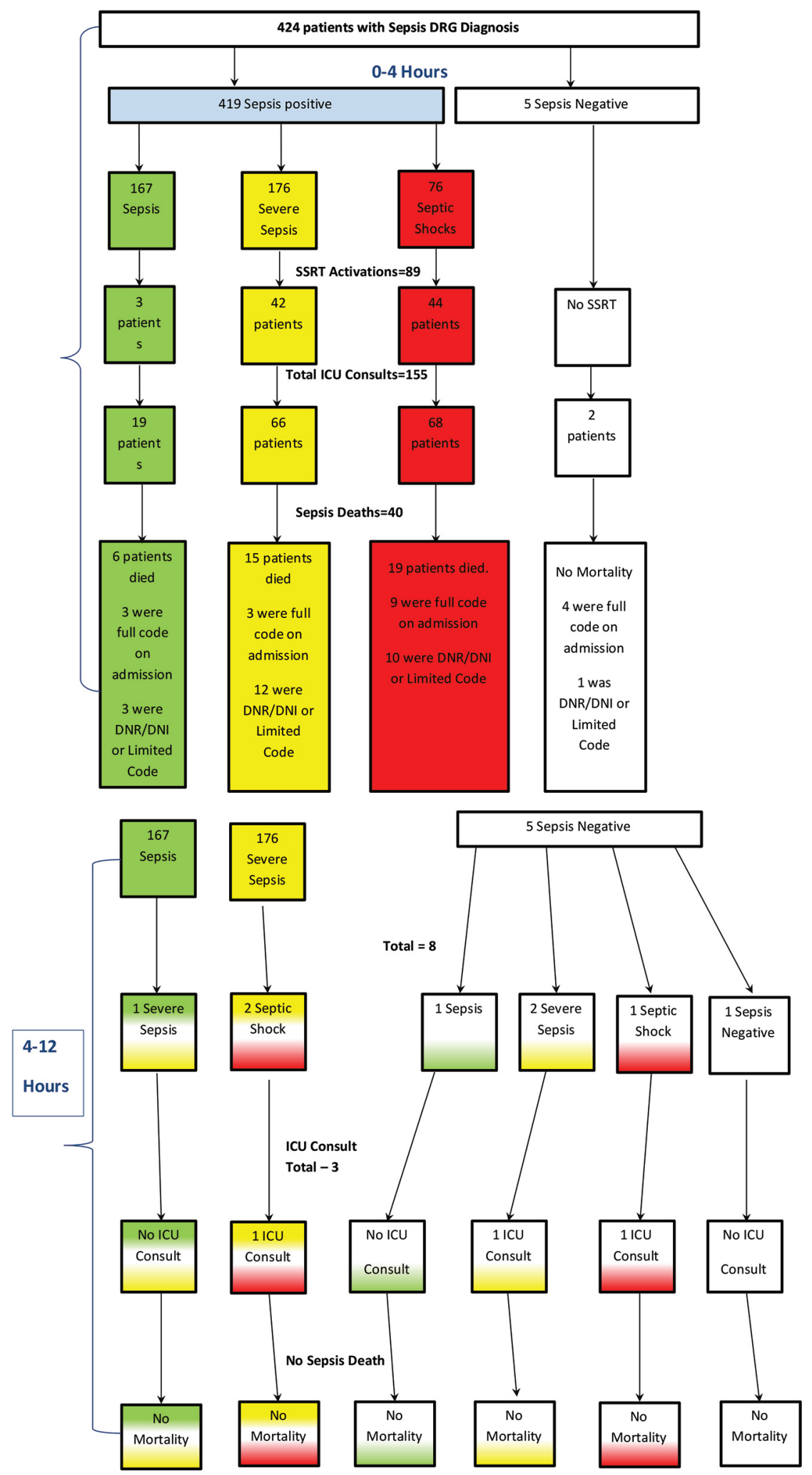

Figure 1. Study Flow chart for Patients Who Were Admitted between September 2013 and August 2014 and had a Sepsis Diagnosis Related Group Code Present upon Discharge, Mayo Clinic Florida. 
Table 3a. Univariate analysis of the whole cohort

\begin{tabular}{lllll}
\hline Patient characteristics & Alive & Dead & Odds ratio & P value $^{\S}$ \\
\hline Number of patients (\%) & $384 / 424(90.6)$ & $40 / 424(9.4)$ & - & - \\
\hline Age, median, IQR & $67.5(55-79)$ & $75(63-89)$ & $0.96(0.94-0.99)$ & $<0.01$ \\
\hline Male sex (\%) & $196 / 219(89.5)$ & $23 / 219(10.5)$ & $0.77(0.40-1.49)$ & 0.51 \\
\hline APACHE IV, median, IQR & $84(72-95)$ & $97(85.5-112.5)$ & $0.96(0.95-0.98)$ & $<0.01$ \\
\hline Full code status (\%) & $317 / 332(95.5)$ & $15 / 332(4.5)$ & $7.89(3.95-15.76)$ & $<0.01$ \\
\hline SSRT activation (\%) & $73 / 89(82)$ & $16 / 89(18)$ & $0.35(0.18-0.70)$ & $<0.01$ \\
\hline ICU Consult (\%) & $125 / 155(80.6)$ & $30 / 155(19.4)$ & $0.16(0.08-0.34)$ & $<0.01$ \\
\hline
\end{tabular}

Table 3b. Univariate analysis for severe sepsis and septic shock cases only

\begin{tabular}{lllll}
\hline Patient Characteristics & Alive & Dead & Odds ratio & P value $^{\S}$ \\
\hline Number of patients (\%) & $218 / 252(86.5)$ & $34 / 252(13.5)$ & - & - \\
\hline Age, median (IQR) & $68(55.8-81)$ & $74(63-89.3)$ & $0.97(0.95-0.99)$ & 0.02 \\
\hline Male sex (\%) & $116 / 135(85.9)$ & $19 / 135(14.1)$ & $0.90(0.43-1.86)$ & 0.85 \\
\hline APACHE IV, median (IQR) & $86(75-100)$ & $98.5(84.8-115)$ & $0.97(0.95-0.99)$ & $<0.01$ \\
\hline Full code status (\%) & $173 / 185(93.5)$ & $12 / 185(6.5)$ & $7.05(3.24-15.32)$ & $<0.01$ \\
\hline SSRT activation (\%) & $70 / 86(81.4)$ & $16 / 86(18.6)$ & $0.53(0.26-1.11)$ & 0.12 \\
\hline ICU Consult (\%) & $107 / 134(79.9)$ & $27 / 134(20.1)$ & $0.25(0.10-0.60)$ & $<0.01$ \\
\hline
\end{tabular}

$\mathrm{ICU}=$ Intensive care unit; IQR=Interquartile range; $S S R T=S$ epsis and shock response team.

${ }^{\S}$ Nonparametric tests (Fisher's and Wilcoxon, as applicable).

Table 4a. Multivariate logistic regression analysis for the whole cohort

\begin{tabular}{lll}
\hline Mortality & Odds ratio & P value \\
\hline APACHE IV & $0.98(0.96-0.99)$ & 0.01 \\
\hline Full code status & $7.71(3.65-16.26)$ & $<0.01$ \\
\hline SSRT activation & $0.69(0.31-1.56)$ & 0.38 \\
\hline ICU consult & $0.22(0.09-0.54)$ & $<0.01$ \\
\hline
\end{tabular}

Table 4b. Multivariate logistic regression analysis for severe sepsis and septic shock cases only

\begin{tabular}{lll}
\hline Mortality & Odds ratio & P value \\
\hline APACHE IV & $0.98(0.96-0.99)$ & 0.03 \\
\hline Full code status & $7.33(3.21-16.74)$ & $<0.01$ \\
\hline ICU consult & $0.26(0.10-0.67)$ & $<0.01$ \\
\hline
\end{tabular}

multivariate analysis; full code status (OR $7.71 ; 95 \%$ CI 3.65-16.26; P value $<0.01^{*}$ ) and ICU consultation on admission (OR 0.22; 95\% CI 0.09-0.54; $\mathrm{P}$ value $\left.<0.01^{\star}\right)$ retained significant association with survival, while SSRT activation consultation did not (OR
0.69; 95\% CI 0.31-1.56; P value 0.38) (Table 4a). In subgroup analysis of patients with severe sepsis/septic shock, SSRT activation did not show a statistically significant association with survival in univariate (OR 0.53 ; 95\% CI 0.26-1.11; P value 0.12) (Tables 3b and $4 \mathrm{~b})$.

The total combined in-hospital mortality of patients with sepsis, severe sepsis, or septic shock was $9.4 \%$ (40/424). Of note, $62.5 \%(25 / 40)$ of expired patients had active Do Not Resuscitate (DNR)/Do Not Intubate (DNI), or limited code at admission (online supplemental material).

\section{Discussion}

This study reports on our academic medical center's experience with the development and implementation of a 2-step machinehuman interface approach to the septic patient in ED, including the CSSA (machine 


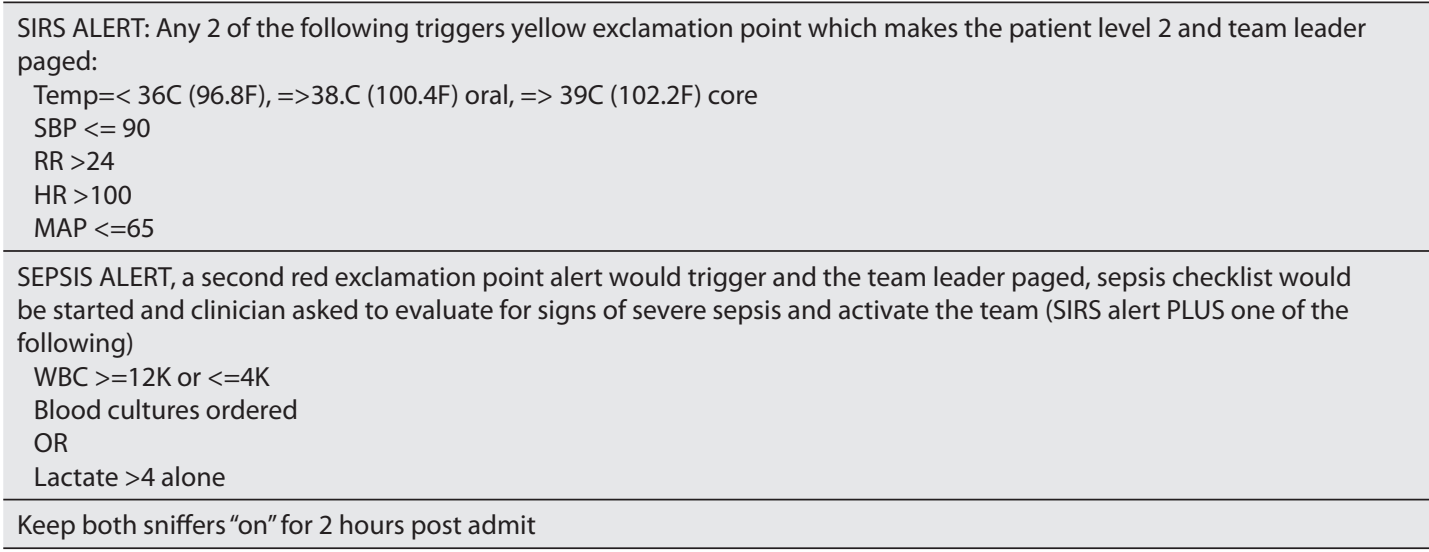

Figure 2. Computerized Sepsis "Sniffer" Algorithm (CSSA)

Algorithm characteristics and cut-off point were derived, tested and refined by the authors at Mayo Clinic Florida in 2013.

algorithm, Figure 2) and the ED physician decision to activate SSRT (human interface).

Evidence suggests that early diagnosis, timely initiation of appropriate antibiotics, and resuscitation to hemodynamic goals, improve clinical outcomes $(4,6,14,17-23)$, while a multidisciplinary approach has been promoted in practice-based guidelines for sepsis management (24). The inability to transform evidence based medicine into clinical implementation has been recognized as one of the great obstacles of modern medicine. Irrespective of improvements in the proper care of critically ill patients, evidence-based interventions continue to be underused (25-29). The barriers consist of resource under-utilization for the critically ill in EDs, poor communication, poor transitions of care, and resistance to multidisciplinary coordination of care. Hewett et al $(30,31)$ have also proposed that the inter-professional and intergroup climate in hospitals leads to poor communication and impedes system approaches to improving healthcare. An intergroup environment in the healthcare facility represents persons identifying with their professional groups, and afterwards, valuing their groups over others, resulting in very poor interaction, unhealthy cooperation, competition for limited resources, and impedance of system standards (31). Types of intergroup members who might be linked to sepsis consist of ED physicians, intensivists, ED nurses, critical care nurses, pharmacists, medical informatics professionals, and hospital administrators. Barriers to implement this project include both the areas of clinical informatics, human factors and implementation science. From the clinical informatics perspective our current EMR does not have the capabilities to send automatic alerts, therefore we have to rely on including another layer of clinical informatics to get the data out of the EMR and run the CSSA algorithms requiring several validation tests. From the human factors and implementation science a significant hurdle we had to overcome was the silos type practice each group had in the $\mathrm{ED}, \mathrm{ICU}$ and hospital service. By developing standardized tools, protocolized ways to communicate and respond depending on sepsis severity were key to overcome these barriers. This project provided a structured setting during which all parties involved in the care of these complex patients could sit together to actively identify and remove barriers to successful implementation of the bundle. Prior to the initiation of the project, the ED and ICU teams were working in silos and each had their own opinion as to who was responsible for which elements of the 
bundle and even which patients required ICU level care. We were able to eliminate this ingrained hospital intergroup tradition by identifying its presence and developing a multidisciplinary SSRT that incorporated team members from almost all key groups coupled with the assignment of respected champions to modify the culture within each individual group. One of the keys to the success of this attempt was to spend time on the front end of the project carefully analyzing key stakeholders and their positions on the elements of the bundle and then painstakingly creating a cohesive team who could agree on triggers for the pager and SSRT activation and, more importantly, create consensus and a spirit of cooperation and teamwork within their respective groups. The creation of the SSRT in particular required buy-in from multiple members of the healthcare team. This strategy enhanced communication and resulted in consensus towards responsibilities among disciplines, where before, obstacles had hindered the administration of treatment protocols.

Utilizing clinical decision support (CDS) systems within electronic health records (EHRs) to improve early identification of septic patients has also been proven beneficial (32-34). Different CDS tools, like the Sepsis Early Alert Tool (SEAT) (32) and the Severe Sepsis Best Practice Alert (SS-BPA) (34) have been evaluated and proven effective as evidenced by decreased time to antibiotic administration in septic patients. The SEAT decreased median time from triage to antibiotic administration by $20 \mathrm{~min}$ utes (32). Also, the SS-BPA, an automated, real time surveillance system for patients meeting sepsis criteria within the EHR, was associated with a higher proportion of patients receiving antibiotics within one hour of diagnosis (34). One hospital sought to develop a sepsis surveillance system that was highly sensitive and specific by initiating an alert only after assessing the patient demographics, vital signs, medications, lab values, discrete documentation elements, medical problems, infectious diagnoses, and signs and symptoms of infection. The novel sepsis screening tool, taking into account an array of factors, reported a sensitivity of $95 \%$ and specificity of $82 \%$ when compared to the gold standard (physician chart review) (33). Despite the fact that current sepsis identification and alert systems have emphasized addressing clinical outcomes, these types of designs have not been successful in providing evidence of benefits in clinically meaningful or patient-centered endpoints $(11,12,31,32)$. Therefore, a better strategy is required to develop and validate a clinically beneficial sepsis alert system, particularly for performance in the ED and critical care environments. Our strategy focused on early recognition through CSSA, which was applicable to all patients presenting to the ED with sepsis. Although our alert system included the same variables as in the study by Brown et al (35), we achieved higher sensitivity with a lower false positive rate (100\% and $4 \%$ versus $80 \%$ and $7 \%$, respectively). A reason for the lower false positive rate compared to Brown et al (35) could be that we adjusted the traditional systemic inflammatory response syndrome alert for respiratory rate and heart rate to 24 breaths/minute and 100 beats/minute, respectively (Appendix 1) to avoid alert fatigue. Our results are also comparable to the results of Manaktala et al (33), with the notable difference that they focused on electronic surveillance of the regular ward patients.

A major goal of this study was to improve the human interface, which was defined as the ED providers' decision to activate the SSRT after the machine interface identified a patient as potentially septic. Based on laboratory values and vital sign criteria, our CSSA was highly sensitive to diagnosing sepsis. Due to lack of advanced algorithms to detect severe sepsis; we rely on human 
factor as the main driver of identification of severe sepsis and decision to activate SSRT. Based on human decision, the SSRT was appropriately utilized in $34 \%(86 / 252)$ and inappropriately in $2 \%(3 / 167)$ of patients. Despite this new expedited care pathway, some of our ED staff continued to use "traditional" critical care consultation (53\%) rather than SSRT activation. This represents a common barrier in healthcare change management, where providers continue to use the more familiar pathways. Whether SSRT underuse is due to under-recognition, disagreement with its use for specific patients, organizational barriers, or a combination thereof, is not clear and would require further investigation. Possible explanations for underuse may be barriers related to physician knowledge or attitudes regarding the SSRT (25). For example, it is possible that physicians failed to recognize that patients were eligible for SSRT activation (e.g., hypoperfusion signs, occult shock). We also found that median APACHE IV score was higher in the group receiving ICU consultation compared to the group receiving SSRT activation (Appendix 1). Therefore, physicians may have decided to implement ICU consultation in more severely ill patients in anticipation of the need for ICU admission. They may have chosen not to use the SSRT in others based on objective data or inherent biases, which we did not set out to measure in our study.

The outcomes of our study suggested that computer mediated screening tools are valuable to identify sepsis patients; however cannot replace clinician's assessment to diagnose severe sepsis. Easily identified, clearcut criteria for sepsis and septic shock as defined by Sepsis-3 are easy to identify with an HER (36). The more subtle criteria that constitute severe sepsis may be missed without astute human assessment and decision to intervene. By using the CSSA we increased the identification of septic patients; with the addition of adding the variable of "human de- cision making" on whether or not to activate the SSRT, we observed sepsis related hospital mortality rate $(9.4 \%)$ which was lower than the reported United States mortality rate for septic patients $(1,37)$. We also observed that our septic shock mortality rates $13.5 \%$ (34 out of 252) are even lower than those reported in ProCESS, ProMISe and ARISE trials (38-40). A fundamental difference is that instead of relying on the human factor recognition of sepsis by a provider or research coordinator in the ED we are utilizing an automated computerized algorithm that helps with earlier identification. The whole premise of earlier resuscitation improving outcomes implies that by identifying earlier and using a dedicated sepsis and shock resuscitation team that might explain why the outcomes achieved are better than expected.

Evaluation of the specific reasons for underutilization of the SSRT would require further study, however, to overcome barriers to SSRT activation we provided educational materials including a provider pocket cards and a SSRT activation flow sheet (Online supplemental material). Additionally, we also implemented simulation training for ED nurses related to sepsis identification, care algorithms, standardized treatment protocols, and clinician roles and responsibilities. This QI supported the results of other studies incorporating a standardized process via education, interdisciplinary patient management, and visual tools, resulting in clinically significant results $(14,15,41)$. Although numerous successful reports on the multidisciplinary approach have been published, limited publications on the human decision to activate sepsis response teams were found.

\section{Limitations}

There are various important limitations to our study. First of all, we recognize that 
pertinent information regarding the complex human algorithm preceding the decision to utilize the SSRT may not have been fully evaluated. Additionally, a cause-andeffect correlation could not be established through our observational research design. Moreover, we did not abstract information on additional essential elements of treatment (e.g., early and appropriate antibiotics) that have an effect on the clinical outcomes of septic patients. An important limitation is that we did not capture what other algorithm management may have been followed if only ICU consultation was used without SSRT activation. We could not ascertain how outcomes were influenced by underuse of the SSRT given confounding variables. Another limitation is the generalizability of our study and applicability of its results. Nevertheless, some of the challenges that we observed are likely to be experienced at other institutions as well. Our sample size was small and therefore firm conclusions about mortality reduction cannot be drawn from this study. Finally, we identified all patients with sepsis using administrative claims data. If we missed any sepsis patients by using this method, this number would likely be very small.

The well-publicized document, Sepsis-3, in February 2016 incorporated the Sequential Organ Dysfunction Assessment (SOFA) score into the diagnosis of sepsis (36). Emergency department providers were resistant to using the SOFA criteria, as it was developed to predict mortality from sepsis, not to diagnose sepsis. The ICU and ED had previously used the same visual algorithm for the SSRT, however, the SOFA criteria was not applicable to the ED setting. Additionally, Sepsis-3 re-defined the terms "sepsis" and "septic shock," while eliminating the term "severe sepsis." "Severe sepsis" was eliminated citing that sepsis by definition is lethal, thus using the term "severe" related to sepsis is repetitive (36). Although the term "severe sepsis" was removed from the spectrum of sepsis, the project team encouraged the term to continue to be utilized in the updated SSRT visual algorithm. This concurrent event may have been a source of persistent confusion with the new algorithm utilizing the retired term.

\section{Conclusions}

Our two-step machine-human interface approach to patients with sepsis utilized an outstandingly sensitive and specific electronic tool followed by more specific human decision-making. As a frequent barrier to early diagnosis and treatment of the septic patient, this approach showed a good balance of sensitivity and specificity, which could allow both better triage and more timely management for different levels of severity of illness while maintaining good outcomes. As with any QI effort, continued education for ED providers would benefit the overall utility of the SSRT resource resulting in improved bundle compliance and patient outcomes. Further research should evaluate provider's reasons for the critical decision of activating versus not activating the SSRT resource.

\section{What is already known on this topic}

Early alerts and prompt management of patient with severe sepsis and septic shock (SS/S) starting in the ED have been shown to improve mortality and other pertinent outcomes. Current published evidence reports a wide variety of cut-off criteria for electronic alerts using data from EHR, and several algorithms for treatment that have been applied in different clinical settings. These published studies endorse a more timely response but fail to consistently report a significant improvement in patient outcomes when applied in non-ICU settings.

\section{What this study adds}

Our study highlights the value of early recognition by a computer algorithm to identify sepsis; the key element is that computer algorithms still lack the ability to correctly diagnose severe sepsis. We improved timely identification of septic patients by using the CSSA. The addition of "human decision making" input, whether or not to activate the SSRT, coincided with the prospective decrease in sepsis related hospital mortality rate, 
which could have been also influenced by other factors, not necessarily related solely to our study activities. We suggest a sequential CSSA followed by the human factor in the form of an experience clinician to assess the patient for severe sepsis yields the best outcomes.

Acknowledgements: The abstract was presented as an oral presentation at the Annual Scientific Meeting of Chest: American college of chest physician, in Montreal, CANADA, October $26^{\text {th }}, 2015$.

Authors' contributions: Conception and design: PMF, EF, VB and MAM; Acquisition, analysis and interpretation of data: PMF, EF, VB, MAM, NAD and ANR; Drafting the article: PMF, EF, VB, MAM, JMN and ELG; Revising it critically for important intellectual content: PMF, EF, VB, MAM, JMN and ELG. Approved final version of the manuscript: VB, EF, MAM, NAD, ANR, ELG, JMN and PMF.

Conflict of interest: The authors declare that they have no conflict of interest.

\section{References}

1. Angus DC, Linde-Zwirble WT, Lidicker J, Clermont G, Carcillo J, Pinsky MR. Epidemiology of severe sepsis in the United States: analysis of incidence, outcome, and associated costs of care. Crit Care Med. 2001;29(7):1303-10.

2. HCUP Facts and Figures: Statistics on HospitalBased Care in the United States, 2009. Rockville, MD: Agency for Healthcare Research and Quality (US); 2011.

3. Kumar G, Kumar N, Taneja A, Kaleekal T, Tarima S, McGinley E, et al. Nationwide trends of severe sepsis in the 21st century (2000-2007). Chest. 2011;140(5):1223-31.

4. Rivers E, Nguyen B, Havstad S, Ressler J, Muzzin A, Knoblich B, et al. Early goal-directed therapy in the treatment of severe sepsis and septic shock. N Engl J Med. 2001;345(19):1368-77.

5. Sebat F, Musthafa AA, Johnson D, Kramer AA, Shoffner D, Eliason M, et al. Effect of a rapid response system for patients in shock on time to treatment and mortality during 5 years. Crit Care Med. 2007;35(11):2568-75.

6. Kumar A, Roberts D, Wood KE, Light B, Parrillo JE, Sharma S, et al. Duration of hypotension before initiation of effective antimicrobial therapy is the critical determinant of survival in human septic shock. Crit Care Med. 2006;34(6):1589-96.

7. Coba V, Whitmill M, Mooney R, Horst HM, Brandt MM, Digiovine B, et al. Resuscitation bun- dle compliance in severe sepsis and septic shock: improves survival, is better late than never. J Intensive Care Med. 2011;26(5):304-13.

8. Bailey TC, Chen Y, Mao Y, Lu C, Hackmann G, Micek ST, et al. A trial of a real-time alert for clinical deterioration in patients hospitalized on general medical wards. J Hosp Med. 2013;8(5):236-42.

9. Jones S, Mullally M, Ingleby S, Buist M, Bailey M, Eddleston JM. Bedside electronic capture of clinical observations and automated clinical alerts to improve compliance with an Early Warning Score protocol. Crit Care Resusc. 2011;13(2):83-8.

10. Nelson JL, Smith BL, Jared JD, Younger JG. Prospective trial of real-time electronic surveillance to expedite early care of severe sepsis. Ann Emerg Med. 2011;57(5):500-4.

11. Sawyer AM, Deal EN, Labelle AJ, Witt C, Thiel SW, Heard K, et al. Implementation of a real-time computerized sepsis alert in nonintensive care unit patients. Crit Care Med. 2011;39(3):469-73.

12. Nguyen SQ, Mwakalindile E, Booth JS, Hogan V, Morgan J, Prickett CT, et al. Automated electronic medical record sepsis detection in the emergency department. PeerJ. 2014;2:e343.

13. Umscheid CA, Betesh J, VanZandbergen C, Hanish A, Tait G, Mikkelsen ME, et al. Development, implementation, and impact of an automated early warning and response system for sepsis. J Hosp Med. 2015;10(1):26-31.

14. Winterbottom F, Seoane L, Sundell E, Niazi J, Nash T. Improving sepsis outcomes for acutely ill adults using interdisciplinary order sets. Clin Nurse Spec. 2011;25(4):180-5.

15. McColl T, Gatien M, Calder L, Yadav K, Tam R, Ong $\mathrm{M}$, et al. Implementation of an Emergency Department Sepsis Bundle and System Redesign: A Process Improvement Initiative. CJEM. 2017;19(2):112-21.

16. Grek A, Booth S, Festic E, Maniaci M, Shirazi E, Thompson K, et al. Sepsis and Shock Response Team: Impact of a Multidisciplinary Approach to Implementing Surviving Sepsis Campaign Guidelines and Surviving the Process. Am J Med Qual. 2017;32(5):500-7.

17. Dellinger RP, Levy MM, Carlet JM, Bion J, Parker MM, Jaeschke R, et al. Surviving Sepsis Campaign: international guidelines for management of severe sepsis and septic shock: 2008. Crit Care Med. 2008;36(1):296-327.

18. Dellinger RP, Levy MM, Rhodes A, Annane D, Gerlach H, Opal SM, et al. Surviving sepsis campaign: international guidelines for management of severe sepsis and septic shock: 2012. Crit Care Med. 2013;41(2):580-637. 
19. Levy MM, Dellinger RP, Townsend SR, LindeZwirble WT, Marshall JC, Bion J, et al. The Surviving Sepsis Campaign: results of an international guideline-based performance improvement program targeting severe sepsis. Crit Care Med. 2010;38(2):367-74.

20. Castellanos-Ortega A, Suberviola B, GarcíaAstudillo LA, Holanda MS, Ortiz F, Llorca J, et al. Impact of the Surviving Sepsis Campaign protocols on hospital length of stay and mortality in septic shock patients: results of a three-year follow-up quasi-experimental study. Crit Care Med. 2010;38(4):1036-43.

21. Kumar A, Ellis P, Arabi Y, Roberts D, Light B, Parrillo JE, et al. Initiation of inappropriate antimicrobial therapy results in a fivefold reduction of survival in human septic shock. Chest. 2009;136(5):1237-48.

22. Gaieski DF, Mikkelsen ME, Band RA, Pines JM, Massone R, Furia FF, et al. Impact of time to antibiotics on survival in patients with severe sepsis or septic shock in whom early goal-directed therapy was initiated in the emergency department. Crit Care Med. 2010;38(4):1045-53.

23. Sivayoham N, Rhodes A, Jaiganesh T, van Zyl Smit N, Elkhodhair S, Krishnanandan S. Outcomes from implementing early goal-directed therapy for severe sepsis and septic shock : a 4-year observational cohort study. Eur J Emerg Med. 2012;19(4):235-40.

24. Dellinger RP, Carlet JM, Masur H, Gerlach H, Calandra T, Cohen J, et al. Surviving Sepsis Campaign guidelines for management of severe sepsis and septic shock. Crit Care Med. 2004;32(3):858-73.

25. Cabana MD, Rand CS, Powe NR, Wu AW, Wilson $\mathrm{MH}$, Abboud PA, et al. Why don't physicians follow clinical practice guidelines? A framework for improvement. JAMA. 1999;282(15):1458-65.

26. Rubenfeld GD, Cooper C, Carter G, Thompson BT, Hudson LD. Barriers to providing lung-protective ventilation to patients with acute lung injury. Crit Care Med. 2004;32(6):1289-93.

27. Kalhan R, Mikkelsen M, Dedhiya P, Christie J, Gaughan C, Lanken PN, et al. Underuse of lung protective ventilation: analysis of potential factors to explain physician behavior. Crit Care Med. 2006;34(2):300-6.

28. Mikkelsen ME, Dedhiya PM, Kalhan R, Gallop RJ, Lanken PN, Fuchs BD. Potential reasons why physicians underuse lung-protective ventilation: a retrospective cohort study using physician documentation. Respir Care. 2008;53(4):455-61.

29. Umoh NJ, Fan E, Mendez-Tellez PA, Sevransky JE, Dennison CR, Shanholtz C, et al. Patient and in- tensive care unit organizational factors associated with low tidal volume ventilation in acute lung injury. Crit Care Med. 2008;36(5):1463-8.

30. Hewett DG, Watson BM, Gallois C, Ward M, Leggett BA. Intergroup communication between hospital doctors: implications for quality of patient care. Soc Sci Med. 2009;69(12):1732-40.

31. Hewett DG, Watson BM, Gallois C. Trust, distrust and communication accommodation among hospital doctors. In: Candlin CN, Crichton J, editors. Discourses of Trust. Houndmills, Basingstoke, Hampshire, UK: Palgrave Macmillan; 2013. p. 36-51.

32. Idrees M, Macdonald SP, Kodali K. Sepsis Early Alert Tool: Early recognition and timely management in the emergency department. Emerg Med Australas. 2016;28(4):399-403.

33. Manaktala S, Claypool SR. Evaluating the impact of a computerized surveillance algorithm and decision support system on sepsis mortality. J Am Med Inform Assoc. 2017;24(1):88-95.

34. Narayanan N, Gross AK, Pintens M, Fee C, MacDougall C. Effect of an electronic medical record alert for severe sepsis among ED patients. Am J Emerg Med. 2016;34(2):185-8.

35. Brown SM, Jones J, Kuttler KG, Keddington RK, Allen TL, Haug P. Prospective evaluation of an automated method to identify patients with severe sepsis or septic shock in the emergency department. BMC Emerg Med. 2016;16(1):31.

36. Singer M, Deutschman CS, Seymour CW, ShankarHari M, Annane D, Bauer M, et al. The Third International Consensus Definitions for Sepsis and Septic Shock (Sepsis-3). JAMA. 2016;315(8):801-10.

37. Kochanek KD, Smith BL. Deaths: preliminary data for 2002. Natl Vital Stat Rep. 2004;52(13):1-47.

38. ProCESS Investigators, Yealy DM, Kellum JA, Huang DT, Barnato AE, Weissfeld LA, et al. A randomized trial of protocol-based care for early septic shock. N Engl J Med. 2014;370(18):1683-93.

39. ARISE Investigators; ANZICS Clinical Trials Group, Peake SL, Delaney A, Bailey M, Bellomo $\mathrm{R}$, Cameron PA, et al. Goal-directed resuscitation for patients with early septic shock. N Engl J Med. 2014;371(16):1496-506.

40. Mouncey PR, Osborn TM, Power GS, Harrison DA, Sadique MZ, Grieve RD, et al. Trial of early, goal-directed resuscitation for septic shock. N Engl J Med. 2015;372(14):1301-11.

41. Na S, Kuan WS, Mahadevan M, Li CH, Shrikhande $\mathrm{P}$, Ray S, et al. Implementation of early goal-directed therapy and the surviving sepsis campaign resuscitation bundle in Asia. Int J Qual Health Care. 2012;24(5):452-62. 